\title{
Alstroemeria esteparica (Alstroemeriaceae) una nueva especie para la flora del Cono Sur de Sudamérica
}

\section{Alstroemeria esteparica (Alstroemeriaceae) a new species to Southern Cone of South America}

\author{
Gloria Rojas ${ }^{1, *} \&$ Carlos M. Baeza ${ }^{2}$ \\ ${ }^{1}$ Museo Nacional de Historia Natural y Museo de la Educación Gabriela Mistral, Casilla 787, Santiago, Chile. \\ ${ }^{2}$ Facultad de Ciencias Naturales y Oceanográficas, Departamento de Botánica, Casilla 160-C, Universidad de Concepción, Concepción, Chile. \\ *E-mail: gloria.rojas@mnhn.gob.cl
}

\section{RESUMEN}

Se hace un análisis morfológico y citotaxonómico de poblaciones de Alstroemeria patagonica Phil., de la estepa patagónica chilena, para aclarar dudas acerca de la variabilidad de esta entidad taxonómica. Para esto, se analizaron 3 poblaciones (Estancia Baño Nuevo, Jeinimeni y Estancia Chacabuco). Se hizo un estudio morfológico detallado en ejemplares recolectados con este fin, más muestras de los herbarios SGO y CONC. Además, se realizó un estudio citotaxonómico de las poblaciones, utilizando los cromosomas como fuente de evidencia taxonómica. Se compararon los resultados con datos de Alstroemeria pygmaea Herb. con el objetivo de esclarecer las diferencias entre estos taxones unifloros y con flores de color amarillo. El estudio da como resultado la presencia de una nueva especie de Alstroemeria para Chile y Argentina.

Palabras clave: Aysén, Chile, estepa, Patagonia.

\section{ABSTRACT}

A morphological and cytotaxonomic analysis of Alstroemeria patagonica Phil. populations is made, from the Chilean Patagonian steppe, in order to clarify questions about the variability of this taxonomic entity. 3 populations were analyzed (Estancia Baño Nuevo, Jeinimeni and Estancia Chacabuco). A detailed morphological study was conducted on field specimens collected for this purpose, plus samples of the SGO and CONC herbaria. In addition, a cytotaxonomic study from collected populations was performed, using the chromosomes as a source of taxonomic evidence. Results were compared with data from Alstroemeria pygmaea Herb., in order to clarify the differences between these unifloral taxa and those with yellow flowers. The study results in the recognition of a new species of Alstroemeria for Chile and Argentina.

Keywords: Aysen, Chile, Patagonia, steppe.

\section{INTRODUCCIÓN}

Tradicionalmente, artículos asociados a alstroemerias de Chile y Argentina han incluido diversos morfotipos provenientes de la Región de Aysén y Magallanes dentro de A. patagonica. Esta situación ha generado que sucesivas descripciones de especies y formas sean asociadas a este taxón, como por ejemplo Alstroemeria nana Rendle (Rendle 1904) y Alstroemeria patagonica Phil. f. biflora Ravenna (Ravenna 1967). A pesar de esto, existe discrepancia en el reconocimiento de estas propuestas, lo que ha derivado en sinonimias generadas en la revisión del género por Bayer (1987) y ratificadas por Sanso

Open Access Journal

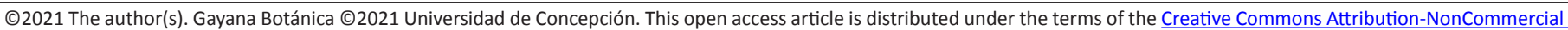
4.0 International License which permits any noncommercial use, distribution, and reproduction, as long as you give appropriate credit to the original author(s) and the source. 
(1996) y Finot et al. (2018). De hecho, Sanso (1996) hace un exhaustivo estudio de taxonomía numérica de este grupo, concluyendo que tanto A. nana como A. patagonica f. biflora son parte de la variación fenotípica de A. patagonica.

Interesantemente, la consistente variación morfológica actualmente observada en ejemplares recolectados en la Región de Aysén sugiere que las variantes morfológicas declaradas dentro de A. patagonica podrían constituir diferentes entidades taxonómicas. En un intento de clarificar esta observación, el presente artículo pretende reportar la variación fenotípica de ejemplares de A. patagonica, las que constituirían evidencia para describir una nueva especie de Alstroemeria para Chile. Para este propósito, se utilizaron ejemplares herborizados de los herbarios CONC y SGO, de modo de comparar y destacar diferencias morfológicas y citotaxonómicas entre $A$. patagonica y el nuevo taxón propuesto. Debido a similitudes de Alstroemeria patagonica con Alstroemeria pygmaea Herb., por ser monoflorales, de pequeño tamaño, casi sésiles y de flores de color amarillo, esta última también fue incluida en este estudio.

\section{MATERIALES Y MÉTODOS}

Las muestras herborizadas consideradas para su análisis (i.e. individuos con flores íntegras y reconocibles) provinieron de varios sectores de la estepa de la Región de Aysén, recolectadas entre los meses de diciembre y enero de los años 2003 al 2017 (Fig. 1). Adicionalmente, se extrajeron muestras vivas desde tres sectores de Aysén para su estudio citológico. Una población con plantas de pequeño tamaño, casi sésiles, proveniente de la Estancia Baño Nuevo, al norte de la ciudad de Coyhaique, la que se consideró como A. patagonica Phil. Las otras dos poblaciones, con individuos con flores de mayor tamaño, provenientes de Quebrada Honda, al lado de la ruta del camino entre Chile Chico y la Reserva Nacional Lago Jeinimeni y muestras de la Estancia Chacabuco (Cochrane), las que se asociaron a la nueva especie.

A partir de los especímenes recolectados y herborizados se midieron diversos parámetros morfológicos. En el caso de las flores, fueron disectadas, fotografiadas y digitalizadas con el software IMAGEJ2 (Rueden et al. 2017). Especímenes de A. pygmaea del norte de Argentina y sur de Bolivia, provenientes de los herbarios LIL y LP, también fueron incluidos en las observaciones morfológicas. Los especímenes recolectados vivos para estudios citotaxonómicos fueron mantenidos y cultivados en el invernadero de Botánica de la Universidad de Concepción.

La metodología citotaxonómica usada consistió en disectar puntas de raíces de 1-2 cm de longitud a partir de material cultivado, las cuales fueron cortadas y pretratadas con una solución de 8-hidroxiquinolina $(2 \mathrm{mM})$ por 24 h a 5 ${ }^{\circ} \mathrm{C}$. Luego, se fijaron en una mezcla de etanol/ácido acético (3:1) durante $24 \mathrm{~h}$. Se realizaron aplastados de puntas de raíces, previo a una hidrólisis ácida con $\mathrm{HCl} 0,5 \mathrm{~N}$ por $17 \mathrm{~min}$ a $42{ }^{\circ} \mathrm{C}$. Posteriormente, se lavó el material y se tiñeron las puntas de raíces con orceína acética al $1 \%$. Los cromosomas se fotografiaron en un microscopio Zeiss Axioskop con cámara de video monocromática incorporada. Las placas metafásicas fueron medidas con la ayuda del programa computacional "MicroMeasure 3.3" (Reeves 2001). A partir de 10 placas metafásicas se calcularon los índices de asimetría del cariotipo AsK\% (Arano \& Saito 1980), Syi (Venora et al. 2002), $M_{C A}$ y $C V_{C L}$ (Peruzzi \& Eroglu 2013) y la longitud total de los cromosomas (LTC). Los dibujos fueron realizados por Gloria Rojas V.

\section{RESULTADOS}

Los tépalos analizados evidenciaron que los de A. patagonica son un tercio más pequeños que los de la nueva especie

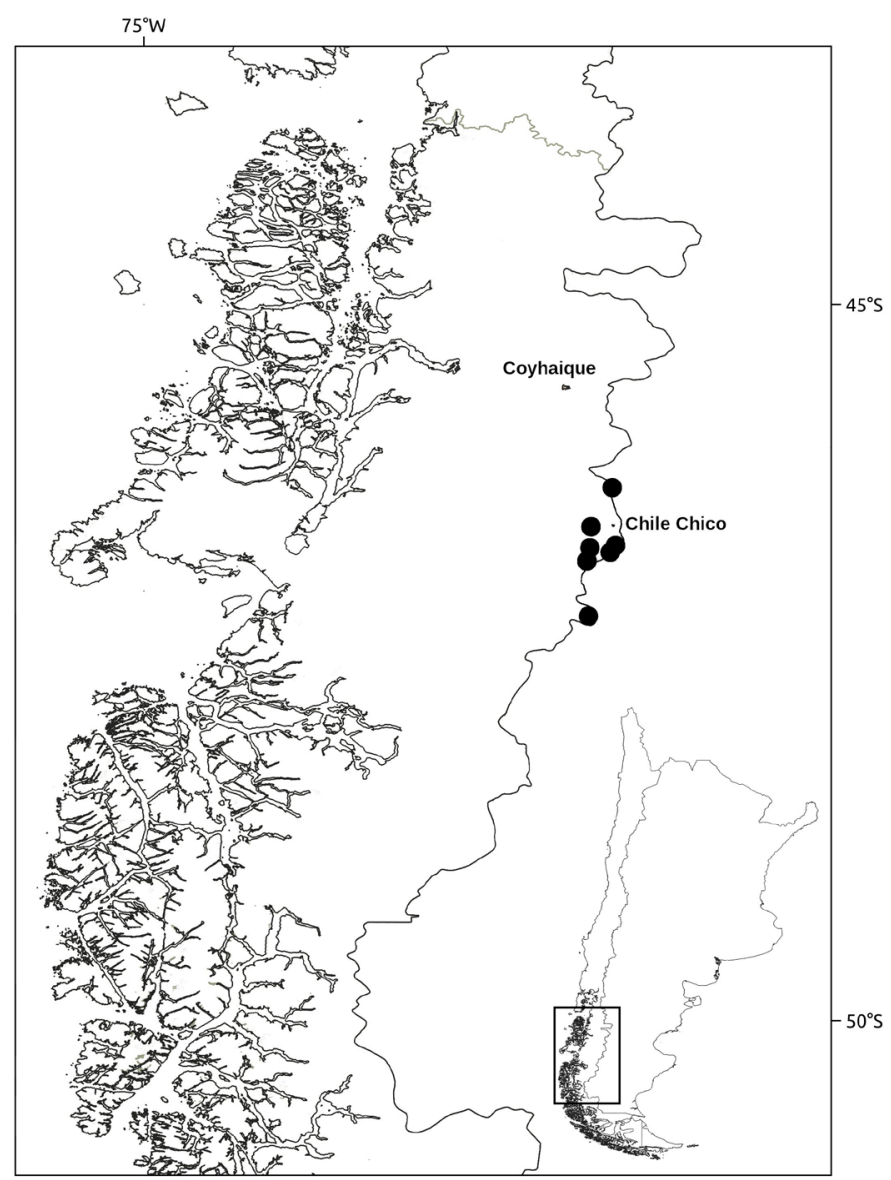

Figura 1. Poblaciones colectadas de Alstroemeria esteparica. / Collected populations of Alstroemeria esteparica. 
(Fig. 2a y 2b). En términos de los caracteres vegetativos, la diferencia en dimensiones puede llegar a ser entre un $300 \%$ a $600 \%$ mayor en la nueva especie que en A. patagonica (Fig. 3). La comparación de las medidas de ambas entidades, en su totalidad, resulta ser de una diferencia de tamaños más que de forma, sobre todo el largo del tallo y el diámetro floral (Tabla 1). Mientras estas observaciones no fueron posibles de cotejar en el material herborizado, la observación directa de ejemplares proveniente de las mismas localidades de colecta confirmó estas y otras diferencias observadas. Por ejemplo, se pudo corroborar en terreno la presencia de diferencias en la simetría floral, las que demuestran que en $A$. patagonica las flores son claramente actinomorfas, comparado a flores zigomorfas presentes en el nuevo taxón (Fig. 4a y 4b).
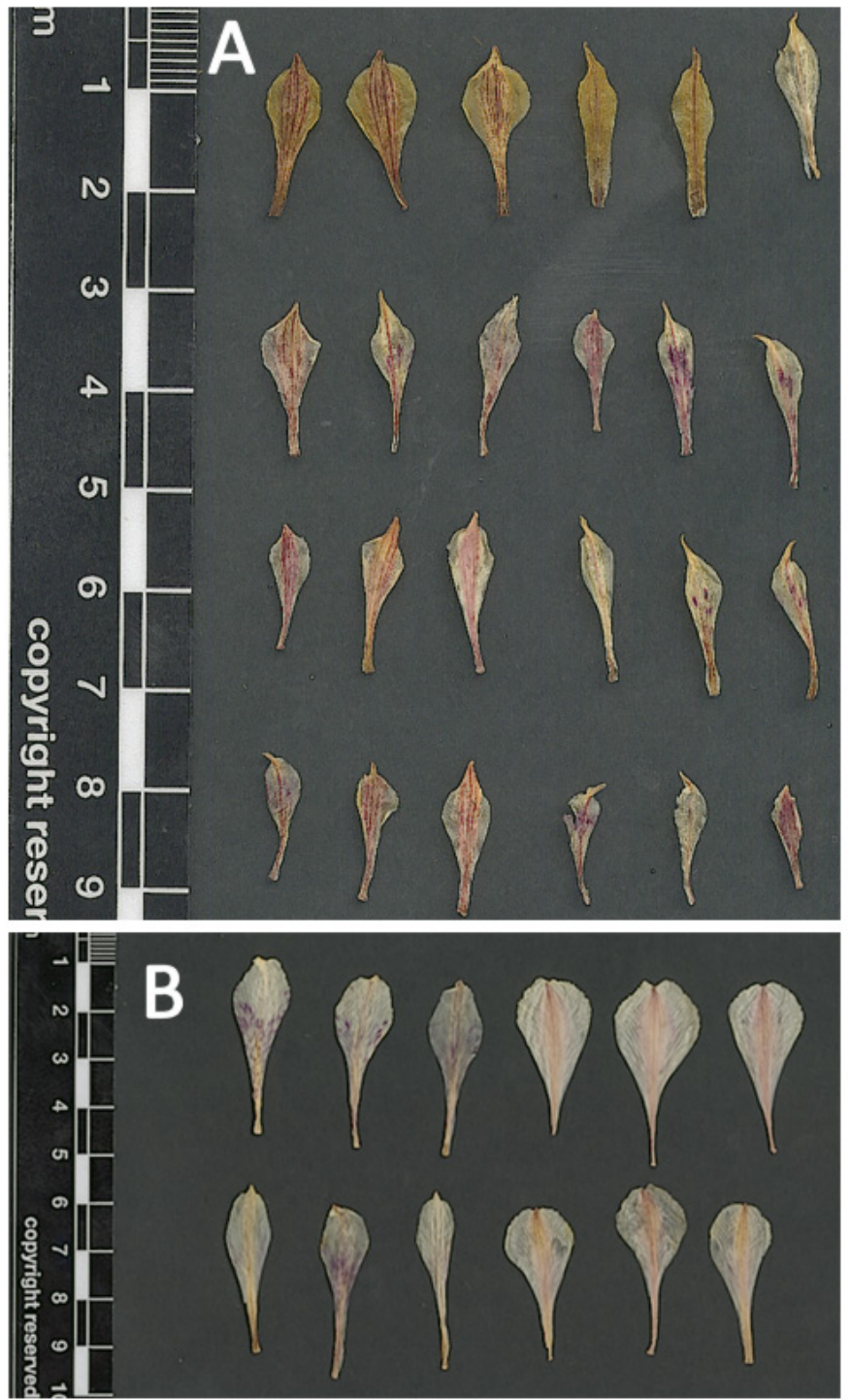

Figura 2. Tépalos: A. Alstroemeria patagonica, B. Alstroemeria esteparica. / Tepals: A. Alstroemeria patagonica, B. Alstroemeria esteparica.

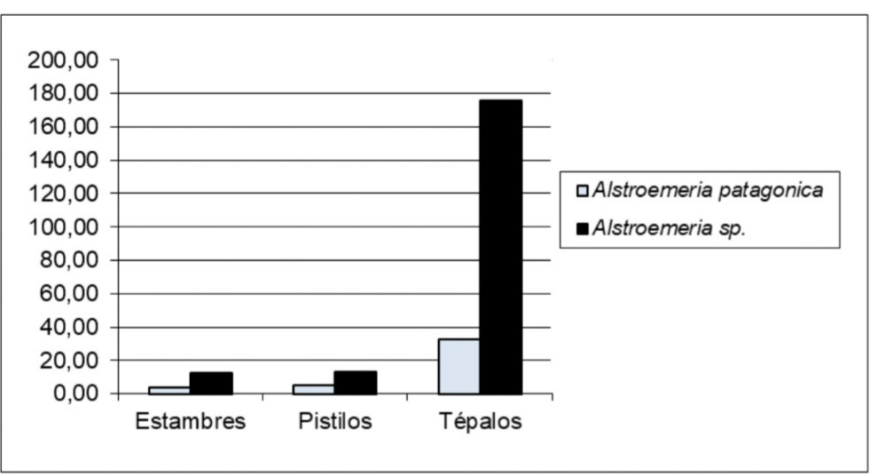

Figura 3. Gráfico comparativo de áreas de verticilos florales de ambas entidades $\left(\mathrm{mm}^{2}\right)$. / Comparative graph of areas of floral whorls of both entities $\left(\mathrm{mm}^{2}\right)$.

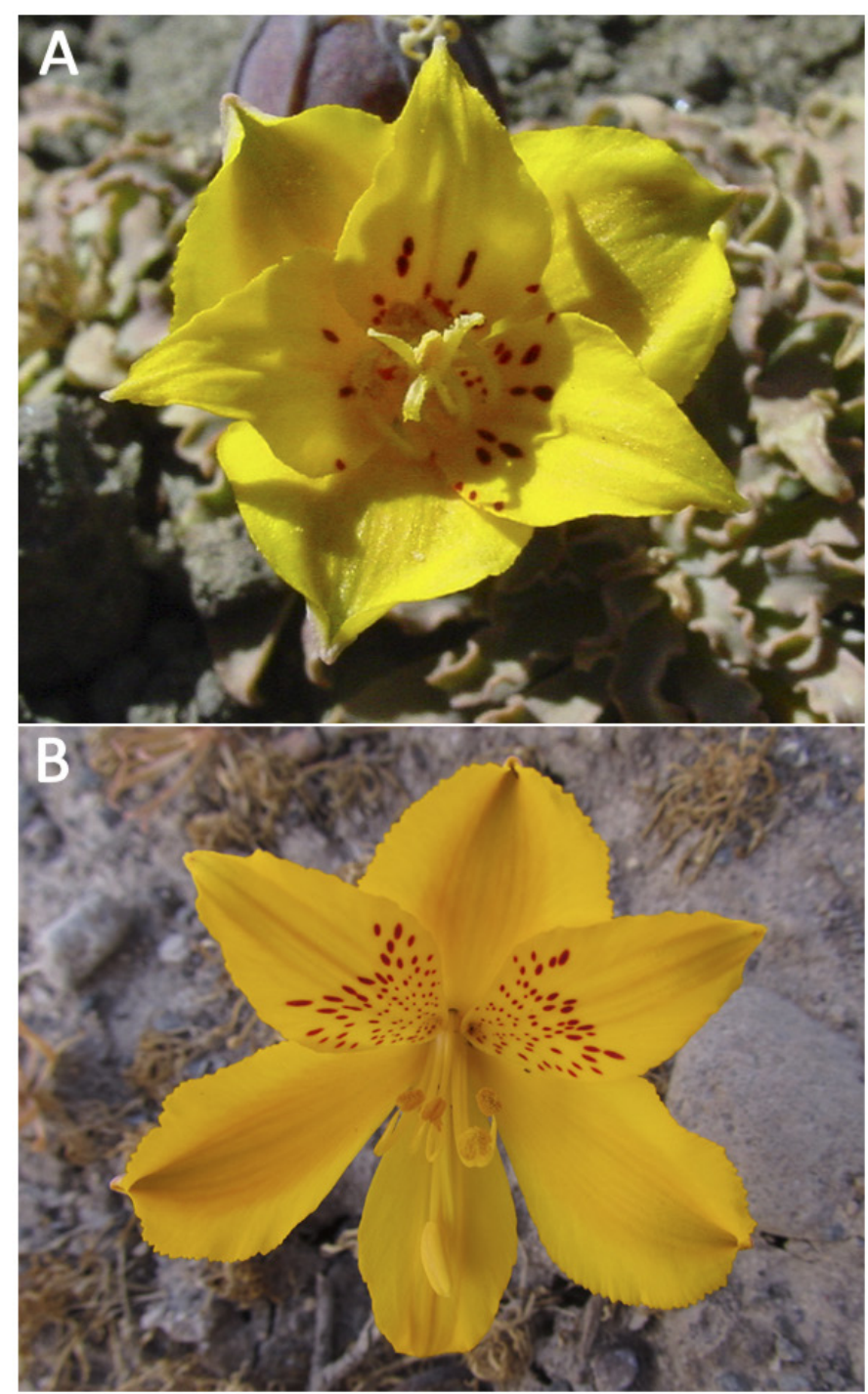

Figura 4. Flores: A. Alstroemeria patagonica, B. Alstroemeria esteparica. / Flowers: A. Alstroemeria patagonica, B. Alstroemeria esteparica. 
TABLA 1. Medidas de los caracteres morfológicos de A. patagonica y A. esteparica. / Measurements of morphological characters of $A$. patagonica and A. esteparica.

\begin{tabular}{lcc}
\hline Caracteres & $\begin{array}{c}\text { A. patagonica } \\
(\mathrm{mm})\end{array}$ & $\begin{array}{c}\text { A. esteparica } \\
(\mathrm{mm})\end{array}$ \\
\hline Altura tallo & 5 & $100-150$ \\
Forma de la hoja & Cinta ondulada & Cinta ondulada \\
Largo de las hojas & 20 & $50-60$ \\
Ancho de las hojas & 2 & $3-5$ \\
Largo tallo & $1-5$ & $20-100$ \\
Largo tépalos calicinales o externos & $13-17$ & $32-35$ \\
Ancho tépalos calicinales o externos & $6-8$ & $8,8-14$ \\
Largo tépalos corolinos o internos & $10-17$ & $32,5-34,5$ \\
Ancho tépalos corolinos o internos & $4-6$ & $8,8-11$ \\
Diámetro flores & $15-20$ & $40-70$ \\
Largo estilos & $10-12$ & $18-25$ \\
Largo estigmas & 2 & $5,8-7$ \\
Largo estambres & $10-13$ & $18-26$ \\
\hline
\end{tabular}

Con respecto a la ecología de ambos taxones, los individuos de A. patagonica se encuentran en cumbres con exposición a vientos provenientes del este, entre guijarros sin vegetación, en suelo compactado y muy desprotegido, solo cercanos a Azorella monantha Clos (Fig. 5a). Al contrario, los individuos del nuevo taxón siempre están entre el matorral, protegidos en laderas y en suelo ligeramente suelto, acompañada por Azorella prolifera (Cav.) G.M. Plunkett \& A.N. Nicolas, Acaena pinnatifida Ruiz et Pav., Berberis buxifolia Lam., Colliguaja integerrima Gillies et Hook., y Festuca pallescens (St.-Yves) Parodi, entre otras (Fig. 5b).

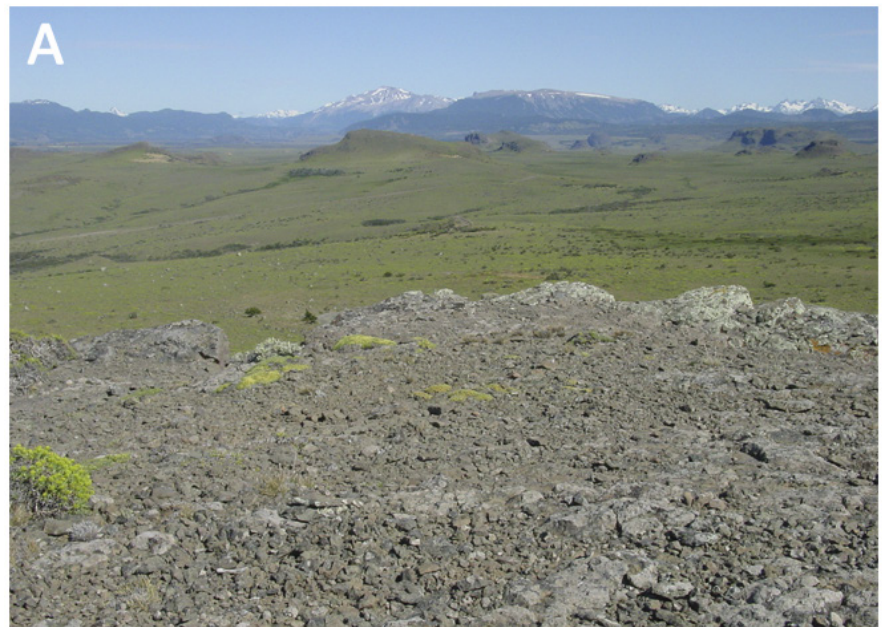

Los resultados del análisis citotaxonómico de ambos taxones evidencian importantes diferencias en términos de asimetría cromosómica, entre los ejemplares identificados como A. patagonica y la nueva especie de Alstroemeria (Tabla 2, Figs. 6 y 7). Ambas especies presentan una fórmula cariotípica muy diferente, que se ve reflejada en los índices de asimetría analizados. La longitud total de los cromosomas es muy superior en A. patagonica y en el gráfico de dispersión se observa que tanto el $\mathrm{CV}_{\mathrm{CL}}$ como el $\mathrm{M}_{\mathrm{CA}}$ contribuyen a separar ambos taxones.

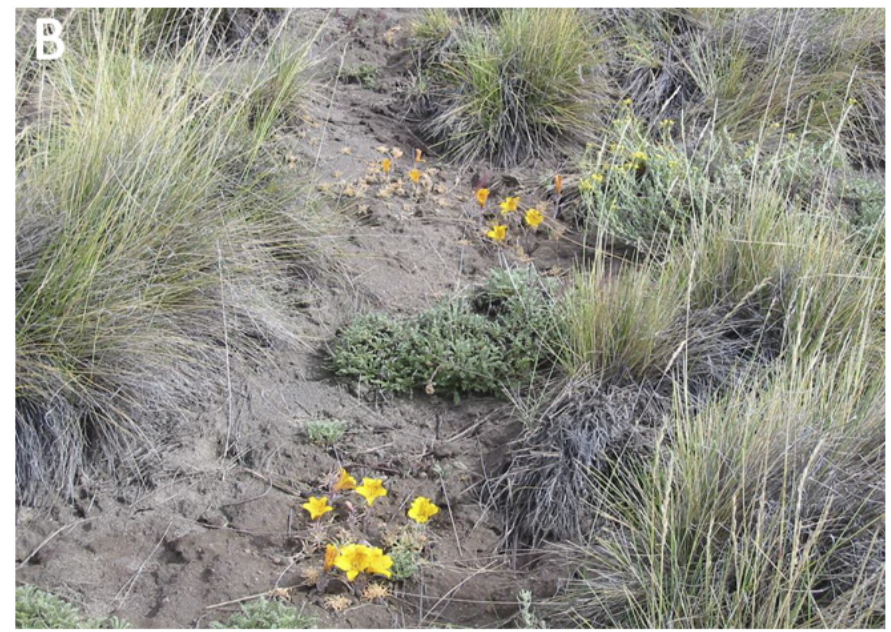

FIgURA 5. Hábitat: A. Alstroemeria patagonica, B. Alstroemeria esteparica. / Habitat: A. Alstroemeria patagonica, B. Alstroemeria esteparica. 
TABLA 2. Comparación de los cariotipos de A. patagonica y A. esteparica. / Karyotype comparation between A. patagonica and A. esteparica.

\begin{tabular}{lcc}
\hline Características del cariotipo & A. patagonica & A. esteparica \\
\hline $2 \mathrm{n}$ & 16 & 16 \\
Fórmula cariotípica & $1 \mathrm{~m}+2 \mathrm{sm}+1 \mathrm{sm}$-sat $+2 \mathrm{st}+1 \mathrm{st}-\mathrm{sat}+1 \mathrm{t}$ & $2 \mathrm{~m}+2 \mathrm{sm}+4 \mathrm{t}$ \\
$\mathrm{LTC}(\mu \mathrm{m})$ & $128,4 \pm 6,5$ & $108,6 \pm 8,2$ \\
AsK $\%$ & $76,0 \pm 3,2$ & $71,2 \pm 2,6$ \\
Syi & 31,5 & 40,4 \\
$\mathrm{CV}$ & $32,04 \pm 1,01$ & $54,5 \pm 0,46$ \\
$\mathrm{M}_{\mathrm{CA}}$ & $55,5 \pm 0,82$ & $50,6 \pm 0,42$ \\
\hline
\end{tabular}

\section{DISCUSIÓN}

Se consideran válidas las observaciones de Bayer (1987) y Sanso (1996), para dejar en la sinonimia a A. nana y A. patagonica $f$. biflora. Sus argumentos son: el espécimen tipo de Alstroemeria nana tiene ejemplares recolectados en diferentes lugares, en la carpeta de herbario se observa que los ejemplares estériles están en un lado y los con flores en otro (Sanso 1996).

Los resultados observados soportarían la existencia de diferencias consistentes entre variantes fenotípicas en Alstroemeria patagonica, las que podrían no estar apropiadamente contextualizadas en la descripción original de la especie. Esto, debido a problemas de interpretación en los rangos de variación de sus caracteres y su falta de asociación a los respectivos ejemplares tipos. Por ejemplo, en nuestro estudio, se identificaron como Alstroemeria patagonica a las plantas de menor tamaño por tener más coincidencia con el tipo de Philippi y por la descripción del hábitat que hizo el recolector Enrique Ibar el año 1877 (Pisano 1977). Philippi (1896), al describir A. patagonica, basado únicamente en un ejemplar de herbario, no logra corroborar sus observaciones desde ejemplares en terreno, él mide el tallo subterráneo, generando confusión en su descripción alusiva al tamaño real de estas plantas (i.e., "tiene un tallo de $85 \mathrm{~mm}$ ").

El presente estudio logra aclarar esta situación, al confirmar que el tallo se encuentra debajo de las hojas y del fruto, los que se sitúan a ras de suelo, demostrando un menor tamaño que el asumido en el ejemplar tipo.

El estudio citotaxonómico revela que los cariotipos de ambas especies son muy disímiles en todos los parámetros, tanto cualitativos como cuantitativos. Baeza et al. (2011) describen el cariotipo de A. patagonica y al comparar esos datos con lo encontrado en la población de Jeinimeni, las diferencias son muy claras (Fig. 6). Además, los índices de asimetría del cariotipo, la longitud total de los cromosomas y la fórmula cariotípica de ambas poblaciones son muy distintas (Tabla 2), lo que también se ve reflejado en el diagrama de dispersión considerando los índices $\mathrm{CV}_{\mathrm{CL}}$ y $\mathrm{M}_{\mathrm{CA}}$ (Fig. 7).

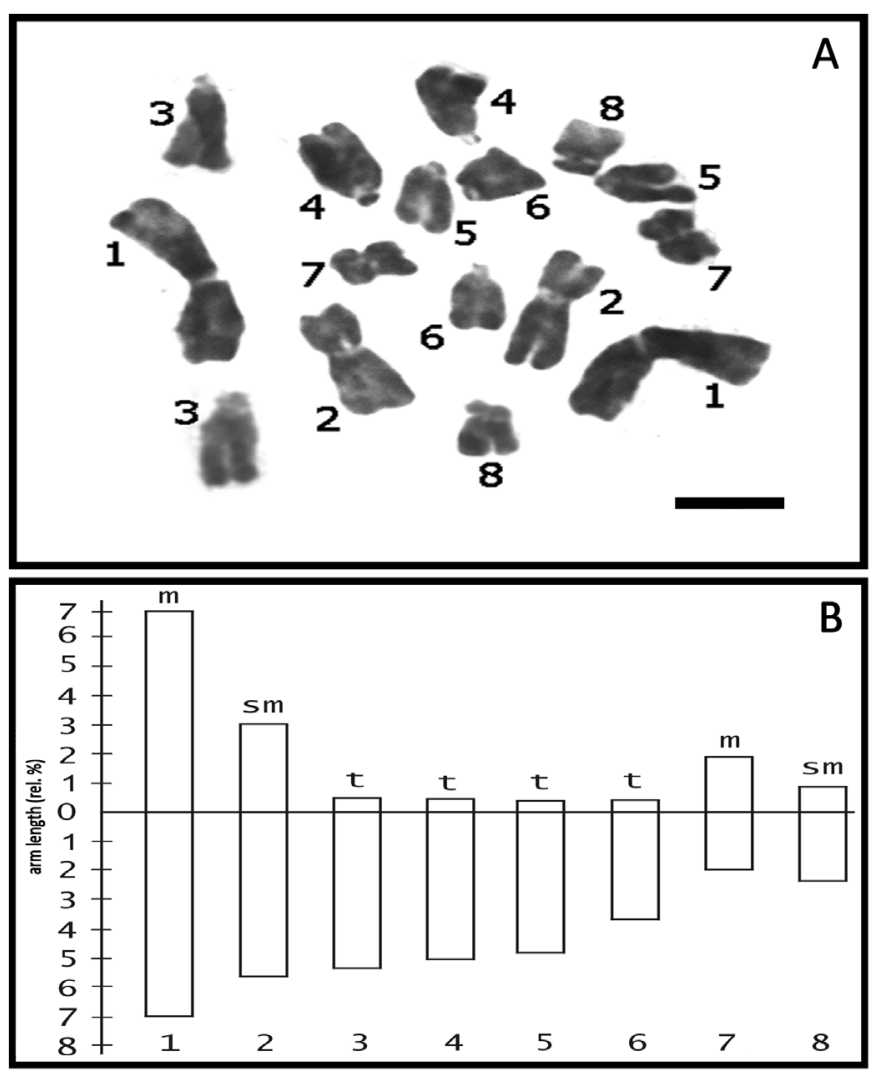

Figura 6. A. Placa metafásica de Alstroemeria esteparica, B. Idiograma de Alstroemeria esteparica. Escala $=10 \mu \mathrm{m} . / \mathrm{A}$. Metaphase plate of Alstroemeria esteparica, B. Idiogramm of Alstroemeria esteparica. Scale $=10 \mu \mathrm{m}$. 


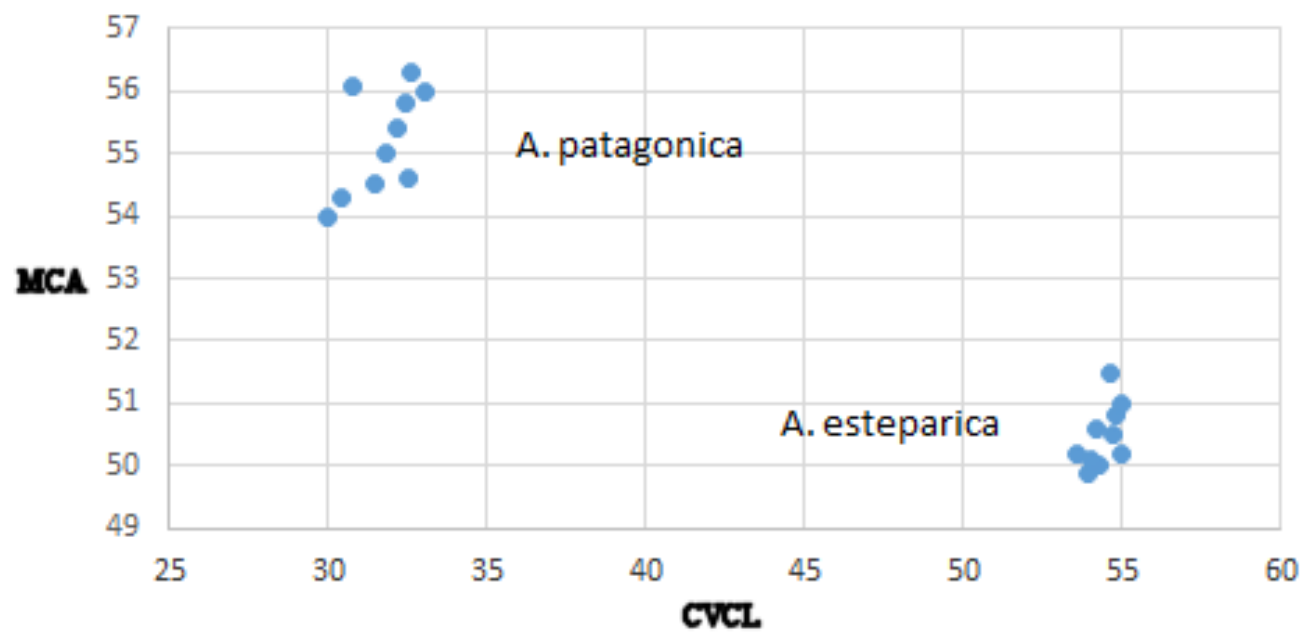

Figura 7. Gráfico de dispersión entre metafases de Alstroemeria patagonica y Alstroemeria esteparica usando valores de $\mathrm{CV}_{\mathrm{CL}} \mathrm{vs} \mathrm{M}_{\mathrm{CA}} /$ Scatter plot among metaphases of Alstroemeria patagonica and Alstroemeria esteparica using values of $\mathrm{CV}_{\mathrm{CL}}$ vs. $\mathrm{M}_{\mathrm{CA}}$.

Considerando los antecedentes morfológicos, ecológicos y citotaxonómicos expuestos, se confirma que, dentro de las alstroemerias presentes en la estepa patagónica, existe más de una especie, razón por la cual se describe un nuevo taxón.

\section{TRATAMIENTO TAXONÓMICO}

Alstroemeria esteparica Gl. Rojas sp. nov.

Tipo: Región de Aysén, Provincia General Carrera, Chile Chico, Quebrada Honda, a $20 \mathrm{~km}$ al Sur de Chile Chico. 739 m (19²7,8'139,0" S- 48¹4,1'54,0" O). 14-I-2003. G. Rojas 6. Typus: Holotipo SGO 169065, Isotipo CONC.

Diagnosis: Distinct by its solitary perianths from all other yellow-flowered Alstroemeria taxa except A. pygmaea and the uniflorus form of sympatric $A$. patagonica. From the former species it differs by its narrower laminas with non-evident venation and plane margins; also by their significant allopatric distributions. The perianth of A. esteparica is zygomorphic and greater than $3 \mathrm{~cm}$ in diameter, that of A. patagonica actinomorphic and less than $2 \mathrm{~cm}$ in diameter. The spotted inner tepal markings of the new species are also notably smaller and more numerous than those of $A$. patagonica.

Hierba perenne, de alrededor de $15 \mathrm{~cm}$ de alto, rizomatosa, con hojas en roseta basal, hojas enteras angostamente lanceoladas de bordes paralelos y ondulados, generalmente carnosas, sin nervadura visible, de $5 \mathrm{~mm}$ de ancho por 50-60 $\mathrm{mm}$ de largo. Tallo de $4-15 \mathrm{~cm}$ de alto, con hojitas escamosas lineares de $1 \mathrm{~cm}$. Flores zigomorfas, unifloras, de color naranjo, con tépalos externos de 3-4 cm de largo por 9-14 mm de ancho, tépalos internos de 3,3-3,5 cm de largo y 9-11 mm de ancho, con 2 ó 3 tépalos con manchas punctiformes, rojizas, más o menos densas. Diámetro de la flor de $7-8 \mathrm{~cm}$, estilo de 1,8-2,5 cm de largo, con estigmas de 0,6-0,7 cm, estambres de 1,8-2,6 cm (Fig. 8a-d). Fruto maduro no observado.

Por lo general, a comienzo de la floración, las hojas estériles están presentes en abundancia en plena floración y en la fructificación ya están secas. Las hojas fértiles suelen ser muy carnosas o coriáceas, las cuales en la medida que el pedicelo floral crece se hacen más delgadas. El fruto suele permanecer según la humedad del ambiente con restos de los estambres y del pistilo adosados a él (Fig. 8b). Las raíces son muy blancas, gruesas y largas, de $1 \mathrm{~cm}$ de diámetro (Fig. 8d).

Etimología: esteparica, de la estepa más austral del cono sur de América, para señalar que es propia de esta formación vegetal.

Ecología y distribución: los ejemplares crecen entre los matorrales y pastizales de la estepa, protegiéndose del viento y se encuentra hacia el sur de Coyhaique desde puerto Ibáñez a la Región de Magallanes.

Redescripción de Alstroemeria patagonica Phil.

Planta en roseta de máximo $2,5 \mathrm{~cm}$ de alto, uniflora. Hojas angostamente lanceoladas de $2 \mathrm{~mm}$ de ancho por $20 \mathrm{~mm}$ de largo. Tépalos muy juntos en la base lo que hace que la flor 

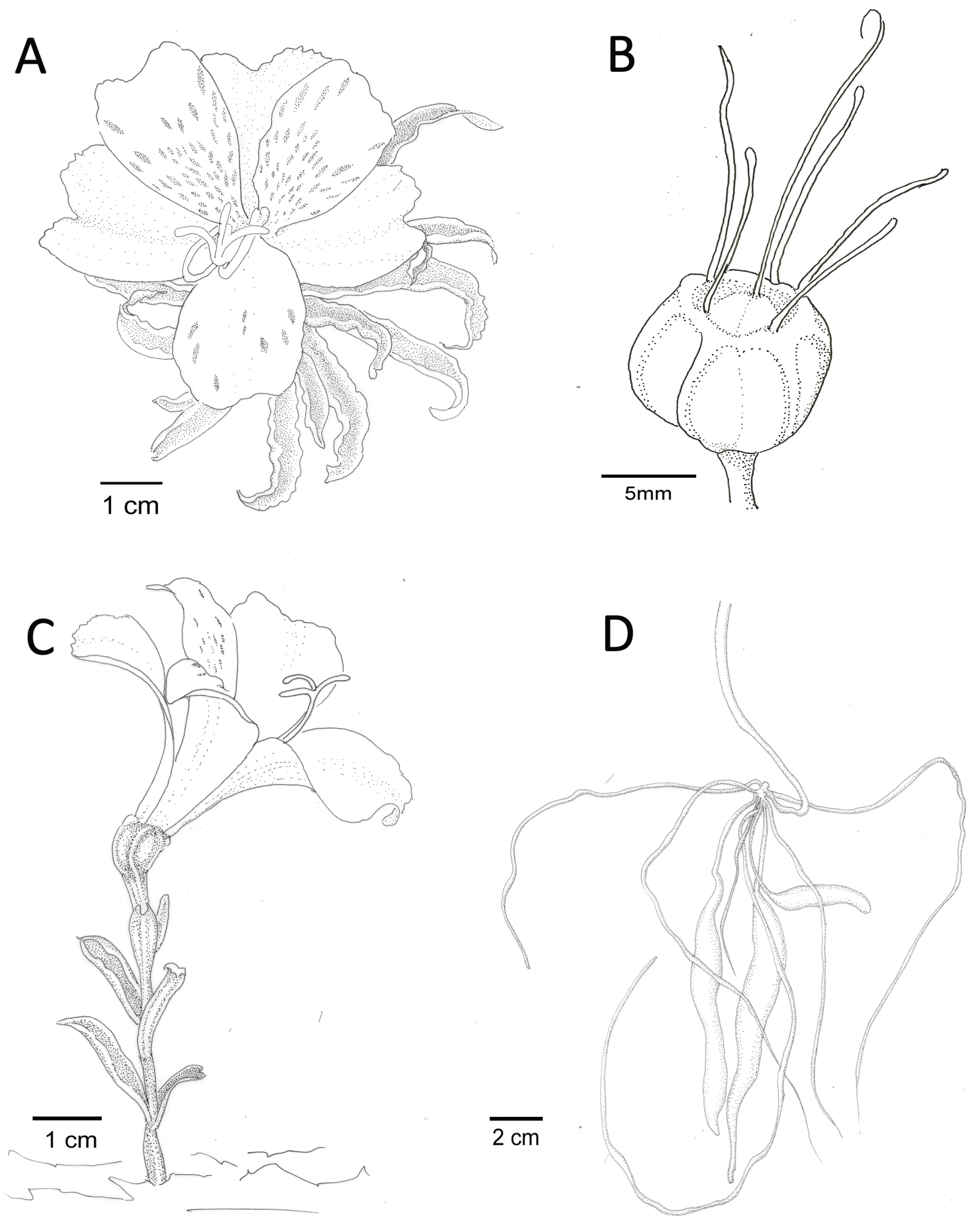

Figura 8. A. Dibujo flor de Alstroemeria esteparica, B. Fruto nuevo con pistilo y restos de estambres de A. esteparica, C. Dibujo del hábito de A. esteparica, D. Raíces almacenadoras de A. esteparica. / A. Flower drawing of Alstroemeria esteparica, B. New fruit with pistil and remains of stamens of A. esteparica, C. Habit of A. esteparica, D. Thickened roots of A. esteparica. 
se asemeje a una trompeta, de simetría radial o actinomorfa, las flores miden entre 1,4-1,7 cm, con un diámetro de la flor menor de $2 \mathrm{~cm}$, estilo de $10-12 \mathrm{~mm}$, estambres de $10-13 \mathrm{~mm}$, fruto sésil. Crece en las cimas de los cerros enfrentando los vientos del Este (Fig. 5a).

Alstroemeria pygmaea Herb.

Descripción adaptada de Hofreiter y Rodríguez (2006).

Planta erecta, desde $1,5 \mathrm{~cm}$ de altura, comúnmente de 4 $\mathrm{cm}$, pudiendo alcanzar los $10 \mathrm{~cm}$ de altura. Tallo rígido, glabro. Hojas lanceoladas, delgadas de 2-5 por 0,5-1 cm, con nervadura notoria. Inflorescencia una umbela erecta con una flor generalmente. Flores de 1-2 cm, erectas, tépalos internos no superiores a los externos, tépalos exteriores amarillos, tépalos interiores espatulados, amarillos, con manchas marrones en el lado interno. Flores zigomorfas. Distribuida desde el centro de Perú hasta Argentina, en altitudes de alrededor de $3500 \mathrm{~m}$.

Es similar a A. patagonica aunque muy diferente de aspecto ya que sus tépalos son sueltos, similares en forma uno de otro, de color amarillo limón, sus hojas son morfológicamente oval-lanceoladas con nervadura notoriamente paralela.

\section{Clave taxonómica de especies de Alstroemerias de color amarillas y unifloras}

A. Hojas lanceoladas, delgadas, pediceladas (base angosta), con nervadura paralela evidente, márgenes lisos. Habita en Perú y el Norte de Argentina por sobre los $3000 \mathrm{msnm}$ Alstroemeria pygmaea A". Hojas angostamente lanceoladas, algo carnosas, no pediceladas, sin nervadura aparente, con márgenes ondulados. Habitan el sur de Chile y Argentina, por debajo de los 1500 msnm

$B^{\prime}$. Flores actinomorfas, menores de $2 \mathrm{~cm}$. Alstroemeria patagonica

$B^{\prime \prime}$. Flores zigomorfas mayores de $3 \mathrm{~cm}$. Alstroemeria esteparica

\section{MATERIALES ESTUDIADOS}

\section{Alstroemeria esteparica}

ARGENTINA. Santa Cruz, Lago Argentino, Tres Banderas, 7-I1964. Garaventa 4573 (CONC 53892).

CHILE. Región de Aysén, Prov. General Carrera, Chile Chico, 230 m. 12-XII-1954. Pfister 4633/7144, (CONC 18466); Chile Chico, Quebrada Honda, 604 m (4647'21.22"S 71'54'24.86"O). 01-II-2005. G. Rojas s.n. (SGO 169067); Chile Chico, Carretera Austral, entre mina Cerro Bayo y Laguna Verde, 414 m (463'6.59"S - 71'52'38.10"O). 11XII-2008. G. Rojas s.n. (SGO 169066); Chile Chico, 594 m

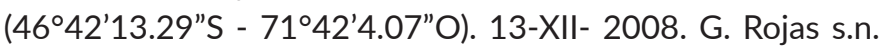
(SGO 169064 y 169063); Chile Chico, $15 \mathrm{~km} \mathrm{S-W}$ de Chile Chico, 800 m (4643'43.52"S - 7144'36.66"O). 07-I-1999. Benyamini 625 (SGO 150113); Chile Chico, Puerto Ibáñez, Laguna Pollolla, 439 m (4640'55.92"S - 71²42'24.83"O). 15XI-2018. D. Penneckamp s.n. (SGO 169646 y 169647); Chile Chico, Río Sucio, $40 \mathrm{~km}$ al sur de Chile Chico, hacia Jeinimeni,

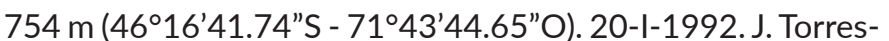
Mura s.n. (SGO 122719); Capitán Prat, Cochrane, Estancia Chacabuco, 612 m (47010'30.00"S - 71'53'47.21"O). 14-XII2008. G. Rojas s.n. (SGO 169068).

\section{Alstroemeria patagonica}

CHILE. Región de Aysén, Prov. Coyhaique, Coyhaique, Ñirehuao, Estancia Baño Nuevo. 740 m (4518'23.13"S 7131'6.61"O). 05-I-2004. G. Rojas 571-1 (SGO 169071); Coyhaique, Ñirehuao, Estancia Baño Nuevo. $742 \mathrm{~m}$ (4518'24.42"S - 71³1'6.89"O). 05-I-2004. G. Rojas 589-2 (SGO 169072); Coyhaique, Ñirehuao, Estancia Baño Nuevo. 743 m (4517'9.24"S - 71'32'23.16"O). 11-I-2004. G. Rojas 569-e (SGO 169073); Coyhaique, Ñirehuao, Estancia Baño Nuevo. 728 m (4517'11.89"S - 71³2'24.80"O). 14-I-2004. G. Rojas 581-f (SGO 169070); Coyhaique, Ñirehuao, Estancia

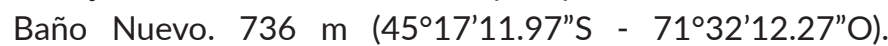
05-I-2004. G. Rojas 588-d (SGO 169069); Coyhaique, Ñirehuao, Estancia Baño Nuevo. 728 m (4517'11.89"S 71'32'24.80"O). 05-I-2004. G. Rojas 581 a (SGO 169074); Coyhaique, Ñirehuao, Estancia Baño Nuevo. $650 \mathrm{~m}$ (451'57.66"S - 71³6'11.48"O). 20-XII-2008. G. Rojas s.n. (SGO 160187); Región de Magallanes, P.N. Pali Aike, Laguna Ana. 110 m (52 ${ }^{\circ} 04^{\prime}$ S-6947' O). 09-I- 2001. Domínguez 2 (CONC 157633); PN Pali Aike, Laguna Ana, sector pastizal, 110 m (52 ${ }^{\circ} 04^{\prime} \mathrm{S}-69^{\circ} 47^{\prime}$ O). 9-I-2001. Domínguez 7400 (CONC 157632); Ultima Esperanza, Sierra de Baguales, Cerro Sta. Lucia, 450 m (5044'S-72 ${ }^{\circ} 20^{\prime}$ O). 03-XII-1984. Arroyo et al. 841053 (CONC 76938); Sector rio de Las Chinas,

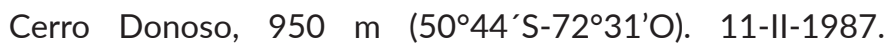


Arroyo et al 870344 (CONC 86556); Última Esperanza, 150 m (5030'S-7400' O). XII-1949. Magens (CONC 11468); Última Esperanza, Sierra de los Baguales, Estancia La Cumbre, Campo La Tropilla, 800 m (5044'S-72²2' O). 17-XII-1986. A. Landero 700 (CONC 93254); Ultima Esperanza, Sierra de Baguales, Cerro Sta. Lucia, 600 m (50 $44^{\prime}$ S- $72^{\circ} 20^{\prime}$ O). 23-XII1984. M. Arroyo 841116 (CONC 76895); Ultima Esperanza, P.N. Torres Del Paine, portería, Sarmiento, $350 \mathrm{~m} \mathrm{(51^{ \circ } 0 1 ^ { \prime } \mathrm { S } -}$ $72^{\circ} 46^{\prime}$ O). 18-XII-2002. D. Rougier 72 (CONC 166209); Última Esperanza, P.N. Torres del Paine, Laguna Amarga. 180 m (5059'S-7245'O). 23-XI-2001. Domínguez 341 (CONC 161828); Ultima Esperanza, Cerro Guido, Estancia Guido, 900 m (5054'S-72 $28^{\prime}$ O). 16-I-1952. Pfister \& Ricardi s.n. (CONC12131); Última Esperanza, Cerro Castillo, $30 \mathrm{~km} \mathrm{~N}$

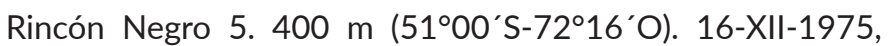
TBPA 635 (CONC 50830); Punta Arenas, San Gregorio. 10 m (52³ $\left.33^{\prime} S-70^{\circ} 15^{\prime} \mathrm{O}\right)$. I-1960. Magens s.n. (CONC 27420).

\section{Alstroemeria pygmaea}

ARGENTINA. Jujuy, Tilcara, arriba de San Gregorio. 3900 m. 27-I-1953. H. Sleumer 3584 (LIL 409401); Jujuy, entre Santos y Ovejería, Nevado Chañi. 3500 m. 9-I- 003. Grau (LIL 606826); Jujuy, Yavi, subida al Abra de Lizoite. $4300-4400$ m. 1-II-1953. H. Sleumer 3673 (LIL 410098); Trancas, El Chorro, en quebrada occidental. 3500-4000 m. XII-1917. R.S. 381 (LIL 30739); Trancas, Cumbre del Chorro, ladera occidental. 3300 m. 1-II-1927. Schreiter 5502 (LIL 30735); Tafi, Peñas azules a Rio de la puerta de San José. 3600-3700 m. XII-1931. Schreiter 7026 (LIL 30738); Jujuy, Tilcara, Pampa Corral. 3800 m. 18-I-1966. Fabris, Crisci y Patriella 6367 (LP); Jujuy, Yavi, Abra de Cajas. 4400 m. 18-I-1966. Cabrera, Malacalsa y von Schmieden 17598 (LP).

\section{AGRADECIMIENTOS}

Se agradece al proyecto Nueva Flora de Chile y al Departamento de Botánica de la Universidad de Concepción, por todas las facilidades otorgadas. A los proyectos Faip, a Isabel Mujica, María Eliana Ramírez, Francisco Mena, Benjamín Molina (Jeinimeni), Patricio Saldivia, Douglas Tomkins, René Mellacura, Cristian Saucedo, Herman Núñez, Juan Carlos Torres y Cristian Berrios.

\section{REFERENCIAS}

Arano, H., Saito, H. 1980. Cytological studies in family Umbelliferae 5. Karyotypes of seven species in subtribe Seselinae. Kromosomo 2: 471-480.

Baeza, C., Rojas, G., Ruiz, E. 2011. El cariotipo fundamental de Alstroemeria patagonica (Alstroemeriaceae). Boletín de la Sociedad Argentina de Botánica 46: 313-315.

Bayer, E. 1987. Die Gattung Alstroemeria in Chile. Mitteilungen der Botanischen Staatssammlung München 24: 1-362.

Finot, V., Baeza, C., Muñoz-Schick, M., Ruiz, E., Espejo, J., Alarcón, D., Carrasco, P., Novoa, P., Eyzaguirre, M. 2018. Guía de campo de las Alstroemerias Chilenas. Corma Editoriales. $295 \mathrm{pp}$.

Hofreiter, A., Rodríguez, E. 2006. The Alstroemeriaceae in Peru and areas vecinas. Revista Peruana de Biología 13(1): 5-69.

Muñoz-Schick, M., Moreira-Muñoz, A. 2003. Alstroemerias de Chile: diversidad, distribución y conservación. Taller La Era, Santiago, Chile. 140 pp.

Peruzzi, L., Eroglu, H. 2013. Karyotype asymmetry: again, how to measure and what to measure? Comparative Cytogenetics 7: 1-9.

Pisano, E. 1977. Contribución de Enrique Ibar Sierra al conocimiento de la naturaleza de la Patagonia Oriental Austral. Vol. 8. Anales del Instituto de la Patagonia, Punta Arenas, Chile.

Philippi, R. 1896. Anales Universidad de Chile 93: 160.

Ravenna, P. 1967. Alstroemeria patagonica forma biflora. Sellowia 19: 34

Reeves, A. 2001. Micro Measure: a new computer program for the collection and analysis of cytogenetic data. Genome 44: 239-443.

Rendle, A. 1904. Alstroemeria nana. Journal of Botany 42: 325.

Rueden, C., Schindelin, J., Hiner, M., DeZonia, B., Walter, A., Arena, E., Eliceiri, K. 2017. ImageJ2: ImageJ for the next generation of scientific image data. BMC Bioinformatics 18: 529. doi: 10.1186/s12859-017-1934-z

Sanso, A. 1996. El género Alstroemeria (Alstroemeriaceae) en Argentina. Darwiniana 34: 349-382.

Venora, G., Blangiforti, S., Ruffini Castiglione, M., Pignone, D., Losavio, F., Cremonini, R. 2002. Chromatin organization and computer aided karyotyping of Triticum durum Desf. cv Timilia. Caryologia 55: 91-98.

Received: 13.08 .2020

Accepted: 17.03.2021 